\title{
Quantitative Analysis of Inclusions in Ductile Iron by Electrolytic Extraction
}

\author{
Fei Yuan ${ }^{1, a}$, Xing Qi ${ }^{1, b}$ and Yitao Yang ${ }^{1, c}$ \\ ${ }^{1}$ School of Materials Science and Engineering, Shanghai University, Shanghai 200072, China \\ ayuanfei@i.shu.edu.cn, b461755018@qq.com, cyangyitao@shu.edu.cn
}

\begin{abstract}
Keywords: ductile iron castings; inclusion; nodularizer; electrolytic extraction
Abstract. Spheroidizing treatment is an important step in the production of ductile iron, in this paper, with the different amount of nodulizer, the morphology and composition of inclusions in molten iron, spheroidized samples and test blocks were studied by scanning electron microscopy (SEM) and energy dispersive spectrometer (EDS), and the inclusions were extracted from the three kinds of samples by electrolytic extraction, XRD analysis was carried out to quantitatively study the effect of residual magnesium content on graphite morphology and spheroidizing rate. The results showed that the amount of inclusions in the molten iron sample was relatively small, whether the amounts in spheroidized samples and test blocks were rather more, and when addition of nodularizer increased from $1.1 \%$ to $1.3 \%$, the total content of inclusions in the castings increased from $0.83 \%$ to $0.93 \%$.
\end{abstract}

\section{Introduction}

In recent years, with the rapid development of wind power, the demand for wind power castings increases continuously, large parts in wind power generation, such as wheels, stands and so on are produced by ductile iron [1]. Castings are usually operated under severe cold and poor conditions, thus the quality of wind power castings is higher than that of ordinary thick ductile iron castings, and it must possess good low temperature toughness [2]. Although the modern refining technology has been considerable developed, it still cannot avoid the existence of inclusions in ductile iron, it is well documented that $[3,4]$, the existence of inclusions could significantly affect the mechanical properties of materials, especially impact toughness. Due to the difference between inclusions and metallic matrix in elasticity and plasticity, during the deformation process, the stress concentrates around the inclusions, which leads to a decreased toughness of materials.

Nodulizer is an essential material for the production of ductile iron, the quality of nodulizer has a direct influence on the quality of ductile iron $[5,6]$, such as spheroidizing rate, graphite ball size and inclusions in castings. The rare earth magnesium silicon nodularizer is commonly used nodulizer, during the nodularizing process, the nodulizer is a certain degree of block. When the nodulizer is in contact with molten iron, the surface begins to melt, of which $\mathrm{Si}, \mathrm{Fe}$ and other atoms enter the molten iron, and $\mathrm{Mg}$ in the $\mathrm{Si}-\mathrm{Mg}$ phase is vaporized into $\mathrm{Mg}$ vapor. These elements combine with the dissolved oxygen in the molten iron to form various inclusions[7], and a part of these inclusions become the nucleation core of graphite, and a small part remains in the cast iron as foreign inclusions.

Electrolytic extraction method is based on the difference of dissolution potential between the cast iron matrix and inclusions in the electrolyte, and the matrix can be dissolved by choosing an appropriate electrolysis conditions, and non-metallic inclusions remain in the anode mud in the form of residues. Inclusions are finally separated by physical method and conducted with a comprehensive study [8]. In this paper, the inclusions of ductile iron samples with different addition of nodulizer were analyzed by the combination of the traditional metallographic preparation method and electrolytic extraction method.

\section{Experimental procedure}

The experimental materials were melted in $150 \mathrm{Kg}$ intermediate frequency induction furnace using high purity pig iron, low carbon steel scrap and returned material as raw materials, the base iron 
sample was took for spectral analysis, then the spheroidizing treatment was carried out. The addition of nodulizer were $1.1 \%, 1.2 \%, 1.3 \%$, corresponding to the furnace number $1,2,3$, and took the nodularized sample for spectral analysis. Stream inoculation technology is followed by, and pouring temperature ranged from 1340 to $1360{ }^{\circ} \mathrm{C}$, unpack temperature is below $300{ }^{\circ} \mathrm{C}$, furan resin sand is used for molding, the test casting blocks were took for inclusions analysis, and the chemical compositions of three groups of test casting blocks are shown in Table 1, it can be seen that the difference of nodularizer addition mainly changes the amount of residual magnesium in test casting blocks.

Table 1 The addition of different amount of nodulizer of three groups of cast test components (wt\%)

\begin{tabular}{cccccccc}
\hline number & $\mathrm{C}$ & $\mathrm{Si}$ & $\mathrm{Mn}$ & $\mathrm{P}$ & $\mathrm{S}$ & $\mathrm{RE}$ & $\mathrm{Mg}$ \\
\hline 1 & 3.71 & 1.98 & 0.12 & 0.025 & 0.020 & 0.016 & 0.040 \\
2 & 3.71 & 1.92 & 0.12 & 0.026 & 0.021 & 0.017 & 0.053 \\
3 & 3.67 & 1.95 & 0.10 & 0.026 & 0.018 & 0.019 & 0.064 \\
\hline
\end{tabular}

The samples were electrolytic extracted by using an electrolytic extractor. The electrolyte was composed of $10 \%$ acetylacetone, $1 \%$ tetramethyl ammonium chloride and anhydrous methanol, the constant current was $0.6 \mathrm{~A}$. After the electrolytic extraction, the products were stood and then centrifuged. The extracted products containing graphite and inclusions need to be separated sequentially based on their different densities, the density of graphite is between 1.8-2.2, while that of $\mathrm{SiO}_{2}$ and other inclusions is above 2.3, thus the flotation separation method[9] was applied. By combining Bromoform dense liquid (Density 2.89) with acetone (density 0.79) according to a certain percentage, we obtained the specific gravity liquid with a density between 2.2-2.3, after standing the extracted products in the specific gravity liquid for $3 \mathrm{~h}$, the products were separated into the double layers, because the density of graphite is smaller, it is reasonable to speculate that it is located in the upper, and the lower is mostly inclusions.

The inclusions were observed and analyzed by tungsten filament scanning electron microscopy (SEM) and its own spectrometer (EDS). The extracted product powders were analyzed by SMART APEXII X-ray diffractometer.

\section{Experimental results}

SEM and EDS analysis of inclusions The inclusions were analyzed by scanning electron microscopy (SEM) and energy dispersive spectroscopy (EDS), respectively, as shown in Fig.1. Due to the different amount of nodulizer addition, the spheroidizing effect of the three groups of cast specimens is different. The state of graphite and inclusions in the sample is analyzed in the non-corroded condition. A class of spherical white inclusions is found in all three groups of specimen, the surface is attached to some irregular shaped inclusions, as shown in Fig 1a. The EDS spectrum displayed in Fig.1b and Fig.1c shows that the spherical inclusions is silicon oxide inclusions, the surface is some composite oxide inclusions . Besides these, kind of black block inclusions are also found in samples, further observation is presented in Fig.1d. The EDS spectrum of the inclusions shows that the inclusions are mainly oxide inclusions of $\mathrm{Mg}, \mathrm{Si}$ and $\mathrm{Fe}$. The nodulizer used in the experiment is rare earth magnesium silicon iron nodulizer which containing elements such as magnesium, rare earth, calcium, and a certain amount of iron, silicon, and a small amount of manganese, aluminum, titanium, etc. According to EDS analysis, it can be speculated that the black inclusions are generated from the addition of nodulizer. 


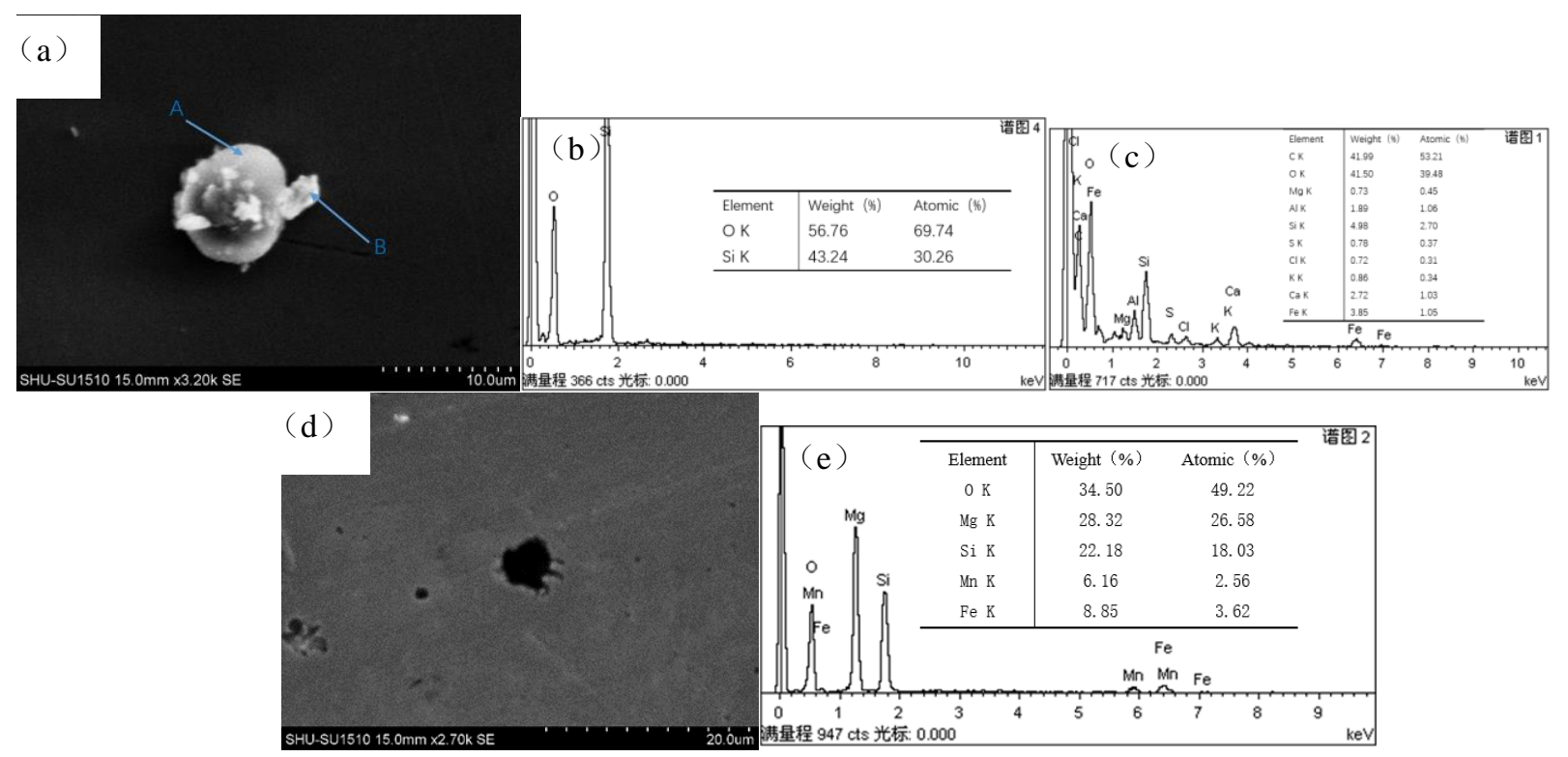

Fig.1 SEM and EDS Analysis of Casting Test Block (a,d)inclusions (b,c,e) inclusions EDS analysis

Twenty fields of view were randomly observed on the samples at a magnification of 100 magnification under a scanning electron microscope, the Image Pro Plus software was performed to statistically analyze the amounts of inclusions, the results is given in Table 2 . It is indicated that the amounts of inclusions in three groups of molten iron sample are nearly the same. According to the EDS analysis, most of the inclusions in the molten iron are $\mathrm{Fe}$ oxide inclusions. After the spheroidizing agent was added during the spheroidizing process, most of the inclusions were floated with the boiling of molten iron[10]. Next step is the addition of slag removal agent for slag process. The floating oxide inclusions are removed, so the total amount of inclusions was significantly reduced. However, the variety of inclusions increases according to the EDS analysis, which is mainly manifested in the increase of $\mathrm{Mg}$-containing oxide inclusions. Before pouring, the fiber asbestos is used to block slag, and the pouring system itself could push off the slag, so the total amount of inclusions is further reduced. Compared with the total amount of inclusions in the three casting test pieces, it can be seen that the addition of nodulant has obvious effect on the inclusions, with the increasing addition of nodulizer, the total amount of inclusions increases correspondingly.

Table 2 Quantitative statistics of inclusions in the molten iron, after the nodulizing and the casting test block (unit $/ \mathrm{mm}^{-2}$ )

\begin{tabular}{c|c|c|c}
\hline amount process & the molten iron & $\begin{array}{c}\text { after the } \\
\text { nodulizing }\end{array}$ & $\begin{array}{c}\text { the casting test } \\
\text { block }\end{array}$ \\
\hline samples & 27 & 18 & 13 \\
2 & 26 & 22 & 18 \\
3 & 27 & 25 & 22 \\
\hline
\end{tabular}

It is difficult to qualify the inclusions by using the statistical method to study the inclusions with some randomness, and only some of the observed inclusions can be studied. In order to further determine the kind and content of inclusions, the inclusions were extracted from the samples by electrolysis, and the extracted products were analyzed.

Analysis of extraction product Firstly, the monolayer extracted product was placed on the conductive adhesive, and the morphological observation and composition analysis were carried out under scanning electron microscope. Fig. 3 is the scanning electron micrograph of the extracted products in the test blocks, and the extracted products were observed and analyzed. In Fig.3a, it can be seen that the graphite is also extracted together with the inclusions. The Figure includes spherical graphite and worm-like graphite, because the proportion of graphite in ductile iron is higher than inclusions, so the graphite in extracted products is rather more, the typical spherical graphite is 
presented in Fig.3b.In addition, a part of inclusions in the worm-like graphite can also be observed. According to the EDS spectrum in Fig.3d, it is shown that the bright white inclusions are oxide inclusions of $\mathrm{Si}$ and $\mathrm{Fe}$. Because the size of worm-like graphite is relatively large, graphite inclusions cannot be observed by optical microscope, which might influence the above statistical results, thus the amount of inclusions observed in No. 1 test block specimen is far lower than the latter two groups of samples.

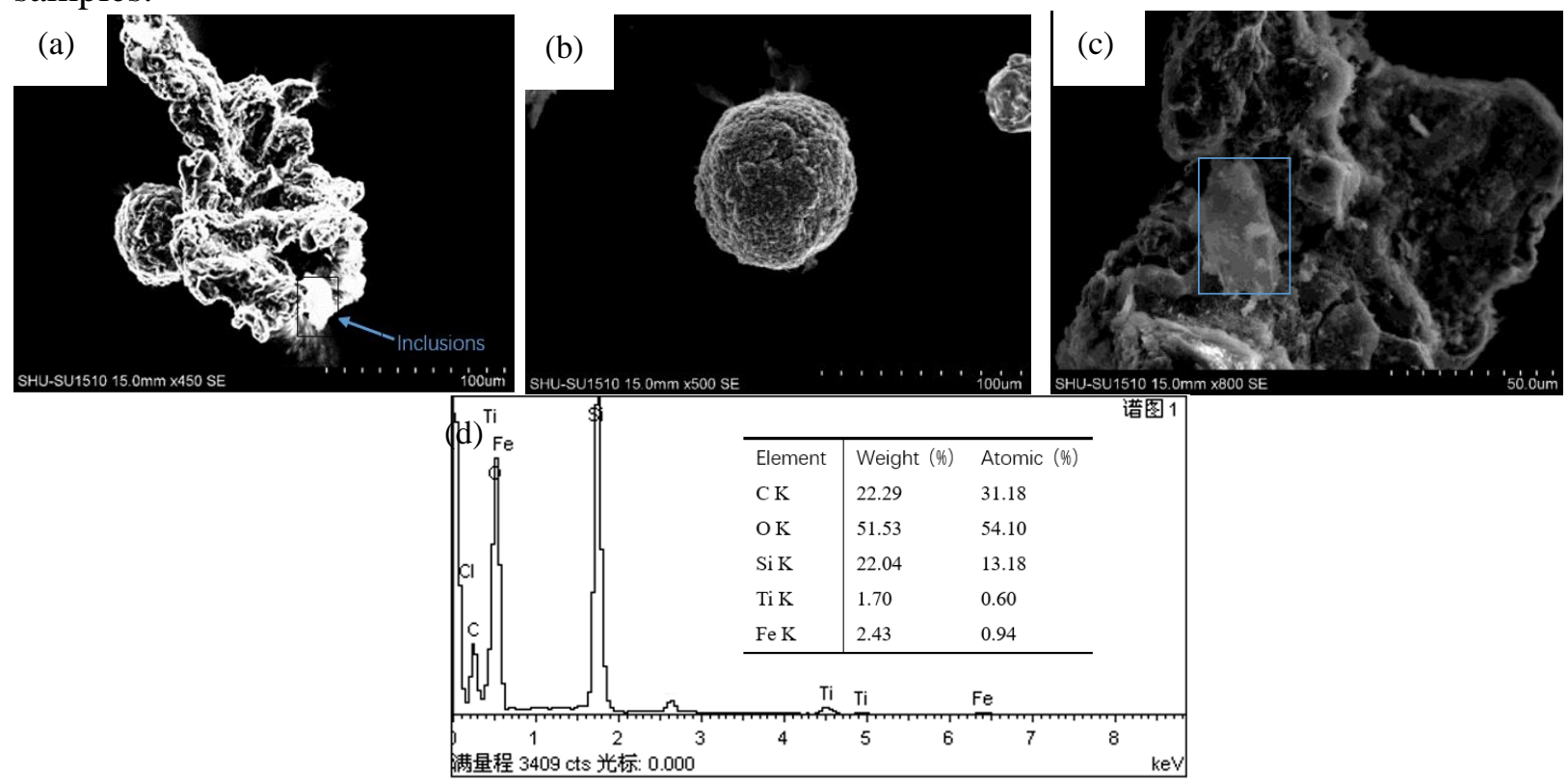

Fig. 3 EDS of extraction products SEM and EDS analysis (a) graphite and inclusions, (b) spherical graphite, (c) inclusion in graphite, (d) inclusion analysis

Most of the graphite and cementite in extracted products are removed after separation, and then the extracted products are qualitative analyzed by XRD. As shown in Fig4, the state of the inclusions in the molten iron samples are close to each other, so No.1 molten iron samples was selected for analysis. In Fig.4a, after separation, there still exists a certain amount of cementite and some graphite, the inclusions in molten iron samples is mainly $\mathrm{SiO}_{2}$ and $\mathrm{FeO}$. After the spheroidization, the extraction products are shown in Fig.4b, compared to the molten iron samples, $\mathrm{Mg}_{2} \mathrm{SiO}_{4}$ and $\mathrm{MgO}$ inclusions increase significantly, magnesium mainly exists in the form of $\mathrm{Mg}_{2} \mathrm{Si}$ in the nodulizers containing magnesium and silicon[11]. $\mathrm{Mg}_{2} \mathrm{Si}$ in inoculant alloy combusts instantaneously in high temperature molten iron, the residue is $\mathrm{MgO}$ and silicon, and silicon further oxidizes to become $\mathrm{SiO}_{2}$ and reacts with $\mathrm{MgO}\left(2 \mathrm{MgO}+\mathrm{SiO}_{2} \rightarrow \mathrm{Mg}_{2} \mathrm{SiO}_{4}\right)$ [12]. According to the observation of peak intensity and area, the inclusions in spheroidized sample 1 are relatively few, and the inclusions in spheroidized sample 3 are the highest.

The XRD spectrum of extracted products in test block samples is shown in Fig. 4c, compared to the spheroidized sample, a different set of peaks appeared around $26^{\circ}$ in the XRD spectrum of the sample c. It can be seen that in the sample c, there is another kind of $\mathrm{SiO}_{2}$ phase, because it appears only in the test block sample, it is speculated that it comes from the molding material (ie, molding sand). The inclusions content in three groups of samples keeps the same with that of the spheroidized samples, and the inclusions content increases with the addition of the nodulizer.

The amount of inclusions can be calculated from the formula: inclusion content $=$ weight of inclusions / (weight of sample before electrolysis - weight of sample after electrolysis). Because extraction products contain graphite and inclusions, the centrifugal, flotation separation are used to remove most of the graphite in the products, and the mass fraction of graphite is calculated alone in the XRD, the total weight of the inclusions is equal to the total weight of the powder minus the weight of graphite. Using the foresaid formula to calculate the content of inclusion, the results can be reached that when the addition of nodulizer is $1.2 \%, 1.2 \%$ and $1.3 \%$, the total content of inclusions in the test block specimen is about $0.83 \%, 0.90 \%$ and $0.93 \%$, respectively. 


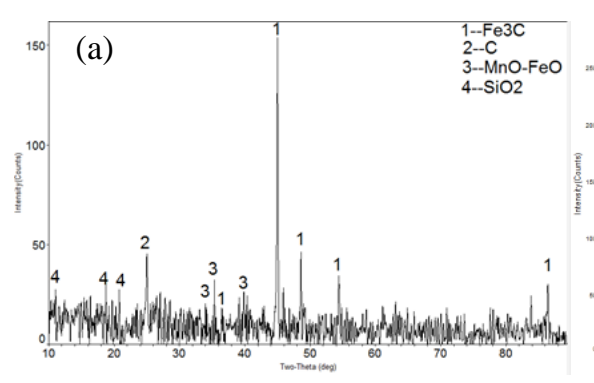

Fig. 4 XRD analysis of the

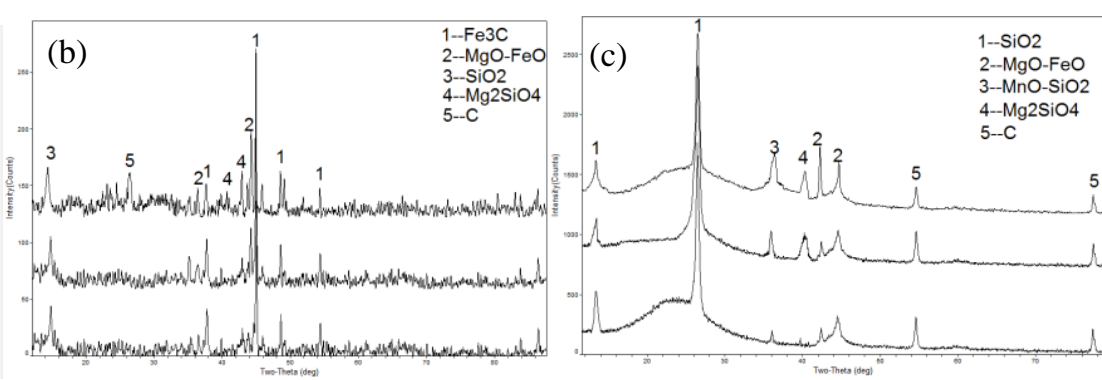

extraction product(a)the molten
spheroidizing, (c)the cast sample

\section{Conclusions}

(1) The results show that there are relatively few inclusions in the molten iron sample, and the inclusions increase obviously after the spheroidizing treatment. With the addition of nodulizer increasing, the inclusions in the sample increases. Compared to spheroidized samples, the inclusions in the final casting are reduced after slagging and casting.

(2) Compared the amount of inclusions in different samples by electrolytic extraction experiment, electron microscopy and XRD analysis, the results show that the variation trend of inclusions contents is close to the statistical results, the more nodulizer is added, the more inclusions such as $\mathrm{MgO}$ and $\mathrm{Mg}_{2} \mathrm{SiO}_{4}$ are formed.

(3) When the addition of nodulizer is $1.1 \%, 1.2 \%$ and $1.3 \%$, the total content of inclusions in cast test blocks is about $0.83 \%, 0.90 \%$ and $0.93 \%$ respectively.

\section{References}

[1] Li P, Fu H, Jiang S. Production Practice of Heavy Nodular Iron Castings Used for Wind Turbine Generator (Chinese). Modern Cast Iron, Vol. 6 (2007), p. 011

[2] Shirani M, Härkegård G. Fatigue life distribution and size effect in ductile cast iron for wind turbine components. Engineering Failure Analysis, Vol. 18 (2011), p. 12

[3] Schmiedt A B, Dickert H H, Bleck W, et al. Evaluation of maximum non-metallic inclusion sizes in engineering steels by fitting a generalized extreme value distribution based on vectors of largest observations. Acta Materialia, , Vol. 95 (2015), p. 1

[4] Wang G, Li S, Ai X, et al. Characterization and Thermodynamics of $\mathrm{Al}_{2} \mathrm{O}_{3}-\mathrm{MnO}-\mathrm{SiO}_{2}(-\mathrm{MnS})$ Inclusion Formation in Carbon Steel Billet. Journal of Iron and Steel Research, International, Vol. 22 (2015), p. 566

[5] Ghahremaninezhad A, Ravi-Chandar K. Deformation and failure in nodular cast iron. Acta Materialia, Vol. 60 (2012), p. 2359

[6] Guo Z T, Sun Y X, Meng L I. Effect of Mg Content in Nodulizer on the Absorptivity of $\operatorname{Mg}($ Chinese). Foundry, Vol. 55 (2006), p. 623

[7] Zhang W H, Wang F, Zhao L S. Formation Reason and Countermeasures of Non-Metal Inclusions of Cast Irons (Chinese). Modern Cast Iron, Vol. 4 (2011), p. 32

[8] Li Y, Sun H, Lan P, et al. Study on non-metallic inclusions extracted by different electrolysis methods in SPHC steel(Chinese). Journal of Wuhan University of Science \& Technology, Vol. 38 (2015), p. 408

[9] Zhang W, Xiong Z, Fang K. The Study of Titanium Inclusions in Grey Cast Iron (Chinese). Foundry Technology, Vol. 3 (1994), p. 46

[10] Hao S. Modern cast iron. (Metallurgical Industry Press, Beijing, 2004).

[11] Zhou H, Huiyong Y U, Sun Y, et al. Effect of FeSiMg $\mathrm{RE}_{2}$ Nodulizer on Microstructure and Property of Ductile Iron (Chinese). Foundry Technology, Vol. 34 (2013), p. 450

[12] YANG Y, XIAO Y. Factors Influencing MgO Content of RE-Mg Nodularizing Alloy (Chinese). Modern Cast Iron, Vol. 3 (2013), p. 15 